\title{
Global Dynamics of an Epidemic Model with a Non-Monotonic Incidence Rate
}

\author{
Seema Khekare ${ }^{1}$, Sujatha Janardhan ${ }^{2}$ \\ ${ }^{1}$ (Department of Applied Mathematics, G.H.Raisoni. Inst. of Engg. \& Tech. for Women, Nagpur, India) \\ ${ }_{2}^{2}$ (Department of Mathematics, St. Francis De Sales College, Nagpur, India)
}

\begin{abstract}
In this paper, we present an epidemiological model with a non-monotonic incidence rate, describing the effect of social phenomenon due to availability of medical infrastructure and awareness of some epidemics, when the number of infective becomes larger. We also analyze the dynamical behavior of the model and derive the stability conditions for the disease-free and the endemic equilibrium. We illustrate theoretical results by carrying numerical simulation.
\end{abstract}

Keywords: Non-monotonic incidence rate, stability conditions, disease-free equilibrium, endemic equilibrium.

\section{Introduction}

The spread of infectious diseases have major universal and economic impact. Social awareness plays an important role to reduce such impacts. Precautionary steps are taken by people due to awareness through education, warning and publicity by the different media such as TV, internet, newspaper, etc. It helps to control the spread of disease and also to reduce the infected individuals.

Sometimes people reported about the disease for few symptoms because of much availability of medical infrastructure which includes hospitals, doctors, medicinal facilities, etc. These people are included in the infected class. But after the diagnosis if the patients are not infected then eventually the number of infected individuals topples down. We can build the models of such infectious disease using mathematical epidemiology which is used to predict the spread and control of the disease.

\subsection{Basic SIR Model}

\section{Basic Concepts Of Epidemiological Dynamics}

The simplest epidemiological model is an SIR model, if individuals recover with permanent immunity. Kermack and Mc Kendrick [1] proposed an SIR model in (1927) for the people infected with a contagious illness in a closed population over time. It explained the rapid rise and fall in the number of infected patients observed in epidemics such as plague and cholera.

Schematically, SIR model can be shown as:

The simplest epidemiological model is an SIR model, if individuals recover with permanent immunity. Kermack and Mc Kendrick [1] proposed an SIR model in (1927) for the people infected with a contagious illness in a closed population over time. It explained the rapid rise and fall in the number of infected patients observed in epidemics such as plague and cholera.

Schematically, SIR model can be shown as:

The simplest epidemiological model is an SIR model, if individuals recover with permanent immunity. Kermack and Mc Kendrick [1] proposed an SIR model in (1927) for the people infected with a contagious illness in a closed population over time. It explained the rapid rise and fall in the number of infected patients observed in epidemics such as plague and cholera.

Schematically, SIR model can be shown as:

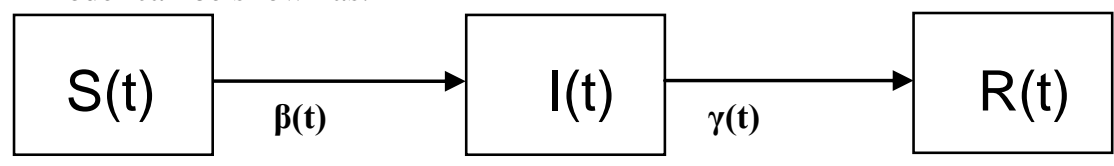

The model consists of a system of three coupled nonlinear ordinary differential equations:

$$
\begin{array}{ll}
\frac{d S}{d t}=-\beta S I & S(0)=S_{0^{p}} \\
\frac{d l}{d t}=\beta S I-\gamma I & I(0)=I_{0^{p}} \\
\frac{d R}{d t}=\gamma I & R(0)=R_{0^{p}}
\end{array}
$$


where $t$ is time, $S(t)$ is the number of susceptible people, $I(t)$ is the number of people infected, $R(t)$ is the number of people who have recovered and developed immunity to the infection, so that $S(t)+I(t)+R(t)=N(t)$ is the total population. $\beta$ is the infection rate, and $\gamma$ is the recovery rate. Also, $S_{0}>0, I_{0}>0 \& R_{0} \geq 0$.

Summing up all the three equations in (2.1.1), we get,

$S^{0}(t)+I^{0}(t)+R^{0}(t)=0$

$\mathbb{N}(t)=S_{0}+I_{0}+R_{0}=\mathbb{N}$

The basic reproduction number $\mathrm{R}_{0}$ is defined as the average number of secondary infections produced when one infected individual is introduced into a population where everyone is susceptible. For the KermackMcKendric model the basic reproduction number is given by,

$R_{0}=\frac{\beta}{\gamma} S_{0}$

If $\mathrm{R}_{0}>1$, then the size of infective class increases and an epidemic occurs. If $\mathrm{R}_{0}=1$, then a diseased individual produces only one new case of the disease and no epidemic can occur. If $\mathrm{R}_{0}<1$, the disease dies out. The basic reproduction number plays a role in public health decisions, because prevention program will be effective in preventing outbreaks only when it ensures $\mathrm{R}_{0} \leq 1$.

An equilibrium of a dynamical system, generated by a system of ordinary differential equations is a solution that does not change with time. If the introduction of a few infective individuals does not result in an epidemic i.e. number of infective tends to zero, then there exist disease free equilibrium. Endemic equilibrium points are steady state solutions where the disease persists in the population.

\subsection{Models with non-monotone incidence rate}

In epidemiology, contact rate is the average number of contacts an individual experiences per unit time. Infection rate is an estimate of the rate of progress of a disease, based on proportional measures of the extent of infection at different time. The incidence rate is the number of new cases per population in a given time period. Standard epidemiological models use a bilinear incidence rate kIS based on the law of mass action. In Hethcote [2], the standard incidence rate is given by $\beta S \mathrm{SI} / \mathrm{N}$, which is the average number of infection transmitted by all infectious individuals, I per day. This works well when there are a large number of susceptible. If the population is saturated with infective, the incidence rate may have a nonlinear dependence on I. Nonlinear incidence rates is of the form $\mathrm{kI}^{\mathrm{p}} \mathrm{S}^{\mathrm{q}}$. If the incidence rate is non-monotone, then it is increasing when I is small and decreasing when I is large. Capasso and Serio [3] introduced a saturated incidence rate $g(1) S$ into epidemic models, where $g(I)$ tends to a saturation level when I gets large, i.e.,

$$
g(D) s=\frac{k I S}{1+a l}
$$

where kI measures the infection force of the disease and $1 /(1+\omega I)$ measures the inhibition effect from the behavioral change of the susceptible individuals when their number increases or from the crowding effect of the infective individuals. This incidence rate is more reasonable than the bilinear incidence rate $g(I) S=$ kIS

because it includes the behavioral change and crowding effect of the infective individuals and prevents the unboundedness of the contact rate by choosing suitable parameters. Ruan and Wang [4] studied an epidemic model with a specific nonlinear incident rate

$g(i) s=\frac{k l^{2} S}{1+\alpha l^{2}}$

and presented a detailed qualitative and bifurcation analysis of the model. The general incidence rate g(i) $S=\frac{k I^{2} S}{1+a l \text { q }}$

was proposed by Liu et al. [5] and used by a number of authors like Hethcote [2], Derrick and van den Driessche [6], Hethcote and Levin [7], van den Driessche [8] and Alexander and Moghadas [9], etc. Xiao and Ruan [10] proposed non-monotone incidence rate

$$
g(D) s=\frac{k l S}{1+a l^{2}}
$$

where $k I$ measures the infection force of the disease and $1+\alpha I^{2}$ describes the psychological or inhibitory effect from the behavioral change of the susceptible individuals when the number of infective individuals is very large. When $\varepsilon=0$, the nonmonotone incidence rate (2.2.2) becomes the bilinear incidence rate (2.2.1). 


\section{Model Formulation}

$\left(1+\mathrm{aI}+\mathrm{bI}^{2}\right)$.

We propose the following epidemiological model with the incidence rate of the form $(1+\mathrm{aI}) \mathrm{kIS} /$

$$
\begin{aligned}
& \frac{d S}{d t}=\alpha-\beta S-\frac{(1+a l) k I S}{1+b I^{2}}+\gamma R \\
& \frac{d l}{d t}=\frac{(1+a l) k I S}{1+b I^{2}}-(\beta+\mu) I \\
& \frac{d R}{d t}=\mu l-(\beta+\gamma) R
\end{aligned}
$$

Where $S(t), I(t)$ and $R(t)$ denote the number of susceptible, infective, and recovered individuals at time $\mathrm{t}$, respectively. $\alpha$ is the recruitment rate of the population, $\beta$ is the natural death rate of the population, $k$ is the proportionality constant, $\mu$ is the natural recovery rate of the infective individuals, $\gamma$ is the rate at which recovered individuals lose immunity and return to the susceptible class, a represents the parameter which measure social phenomenon due to availability of medical infrastructure and $\mathrm{b}$ is the parameters which measure awareness of the people through education and publicity, etc.

We consider the biological meaning of the components $(S(t), I(t), R(t))$ and focus on the model in the first octant of $\mathrm{R}^{3}$. We discuss the existence of equilibriums of system (3.1). For any values of parameters, model (3.1) has a disease free equilibrium $E_{0}=(\alpha / \beta, 0,0)$. We find the positive equilibrium by taking R. H. S. of (3.1) equal to zero.

$$
\begin{aligned}
& \alpha-\beta S-\frac{(1+a l) k I S}{1+b I^{2}}+\gamma R=0 \\
& \frac{(1+a I) k I S}{1+b I^{2}}-(\beta+\mu) I=0 \\
& \mu I-(\beta+\gamma) R=0
\end{aligned}
$$

Then,

$\left[\frac{k a \gamma \mu}{\beta+\gamma}-b \beta(\beta+\mu)-k a(\beta+\mu)\right] I^{2}+\left[a k a-k(\beta+\mu)+\frac{k \gamma \mu}{\beta+\gamma}\right] I+\alpha k-\beta(\beta+\mu)=0$

We define the basic reproduction number as follows:

$R_{0}=\frac{\alpha k}{\beta(\beta+\mu)}$

Then, we have the following:

Theorem 3.1:

(i) if $\mathrm{R}_{0}<1$, then there is no positive equilibrium;

(ii) if $\mathrm{R}_{0}>1$, then there is a unique positive equilibrium $E^{*}=\left(S^{*} I_{0}^{*}{ }^{*} R^{*}\right)$, called the endemic equilibrium.

Endemic equilibrium $E^{*}=\left(S^{*}{ }_{0}{ }^{*}{ }_{a} R^{*}\right)$ is given by

$s^{*}=\frac{\left(1+b I^{*}\right)(\beta+\mu)}{k\left(1+\omega I^{*}\right)}$

$I^{*}=\frac{-\Delta+\sqrt{\Delta^{2}-4 \beta(\beta+\mu)[\beta b(\beta+\mu)(\beta+\gamma)+k a \beta(\beta+\gamma+\mu)]\left(1-R_{0}\right)}}{2[\beta b(\beta+\mu)(\beta+\gamma)+k a \beta(\beta+\gamma+\mu)]}$

$R^{*}=\frac{\mu I^{*}}{\beta+\gamma}$

$\Delta=\mathbb{k}(\beta+\mu)(\beta+\gamma)-\alpha k a(\beta+\gamma)-k \gamma \mu$

The number of infected individuals in endemic state $\mathrm{I}^{*}$ depends on the basic reproduction number $\mathrm{R}_{0}$. If the disease goes to extinct then endemic state will not exist. Bifurcation of steady states and exchange of stability is shown in Fig.1.

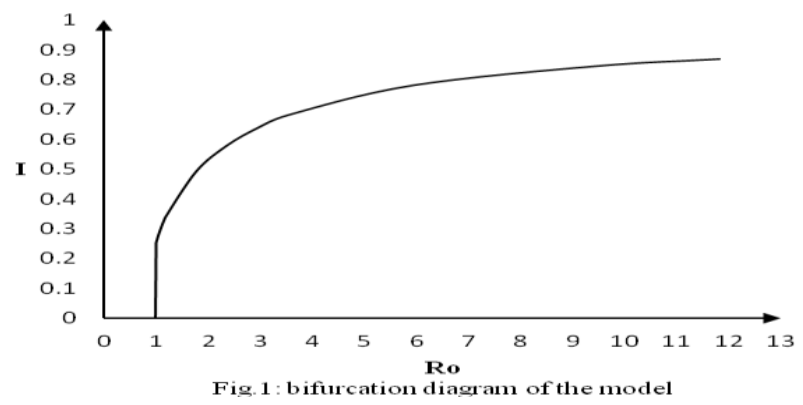




\section{Global Analysis}

In this section, we study the properties of the equilibriums and derive the stability conditions for the disease-free and the endemic equilibrium of model (3.1).

Lemma 4.1: The plane $S+I+R=\alpha / \beta$ is an invariant manifold of system (3.1), which is attracting in the first octant.

Proof: Summing up the three equations in (3.1) and denoting $S(t)+I(t)+R(t)=N(t)$, we have

$\frac{d N}{d t}=\alpha-\beta N$

For the equilibrium point, set

$\alpha-\beta N=0$

From the above equation, it is clear that $\mathbb{N}(t)=\alpha / \beta$ is one solution of eq. (4.1).

The second solution in general is given by,

$N(t)=\frac{\alpha}{\beta}+c e^{-\beta t}$

For any $N\left(t_{0}\right) \geq 0$, the general solution of eq.(4.1), using eq.(4.2), is

$N(t)=\frac{1}{\beta}\left[\alpha-\left(\alpha-\beta N\left(t_{0}\right)\right) e^{-\beta\left(t-t_{0}\right)}\right]$

Also,

$\lim _{t \rightarrow \infty} N(t)=\frac{\alpha}{\beta}$

This completes the proof.

Remark: The limit set of system (3.1) is on the plane $\mathrm{S}+\mathrm{I}+\mathrm{R}=\alpha / \beta$. Thus, we consider the reduced system

$\frac{d l}{d t}=\frac{(1+a l) k}{1+b I^{2}} I\left(\frac{c}{\beta}-I-R\right)-(\beta+\mu) I \equiv P(I, R)$

$\frac{d R}{d t}=\mu I-(\beta+\gamma) R \equiv Q\left(a_{0} R\right)$

We have the following result regarding the nonexistence of periodic orbits in system (4.3), and hence the nonexistence of periodic orbits of system (3.1) using Lemma 4.1.

Theorem 4.2: System (4.3) does not have nontrivial periodic orbits.

Proof: Consider system (4.3) for $I>0$ and $\mathbb{R}>0$. Take a Dulac function

$D(I, R)=\frac{1+b I^{2}}{(1+a l) k l}$

We have

$\frac{\partial(D P)}{\partial I}+\frac{\partial(D Q)}{\partial R}=-1-\frac{(\beta+\mu)}{k}\left[\frac{a b I^{2}+2 b I-\alpha}{(1+a l)^{2}}\right]-\frac{(\beta+\gamma)\left(1+b I^{2}\right)}{k I(1+a I)}$

We consider $y=\mu$

$\frac{\partial(D P)}{\partial I}+\frac{\partial(D Q)}{\partial R}=-1-\frac{(\beta+\mu)}{k}\left\{\frac{a b I^{2}+2 b I-a}{(1+a l)^{2}}-\frac{\left(1+b l^{2}\right)}{l(1+a l)}\right\}$

Solving only the curly bracket, we get

$\frac{a b l^{2}+2 b l-a}{(1+a l)^{2}}-\frac{\left(1+b l^{2}\right)}{l(1+a l)}=\frac{2 b l^{2}+3 b l^{2}+1}{l(1+a l)^{2}}$

As $\mathrm{a}, \mathrm{b}$ are positive, for $\mathrm{I}>0$, this expression is positive. For $\mathrm{I}=0$ it is positive unbounded.

Thus the expression (4.4) remains negative for $\mathrm{I} \geq 0$.

For the general $\gamma$ and $\mu$, expression (4.4) becomes

$\frac{\partial(D P)}{\partial I}+\frac{\partial(D Q)}{\partial R}=-1-\frac{1}{k}\left\{\frac{(\beta+\mu)\left(a b l^{2}+2 b I-\alpha\right)}{(1+a l)^{2}}-\frac{(\beta+\gamma)\left(1+b l^{2}\right)}{l(1+a l)}\right\}$

Expression in curly bracket simplifies to

$$
\begin{aligned}
& \frac{(\beta+\mu)\left(a b I^{2}+2 b I-\alpha\right)}{(1+a l)^{2}}-\frac{(\beta+\gamma)\left(1+b I^{2}\right)}{I(1+a l)} \\
& =\frac{a b I^{2}(2 \beta+\mu+\gamma)+b I^{2}(3 \beta+2 \mu+\gamma)+a l(\gamma-\mu)+(\beta+\gamma)}{I(1+a l)^{2}}
\end{aligned}
$$

which is positive if $\gamma \geq \mu$. Thus the expression (4.4) is negative for $\gamma \geq \mu$.

More general treatment gives 


$$
\begin{aligned}
& \frac{\partial(D P)}{\partial I}+\frac{\partial(D Q)}{\partial R}=-1-\frac{(\beta+\mu)}{k}\left[\frac{a b I^{2}+2 b l-\alpha}{(1+\alpha l)^{2}}\right]-\frac{(\beta+\gamma)\left(1+b l^{2}\right)}{k l(1+a l)} \\
& =-\left\{\frac{I^{2}\left(a^{2} k+2 a b \beta+a b \mu+a b \gamma\right)+I^{2}(2 a k+3 b \beta+2 b \mu+\gamma b)+l(k+\alpha y-a \mu)+(\beta+\gamma)}{l(1+a l)^{2}}\right\}
\end{aligned}
$$

So if $\mathrm{k}$ is such that $k+\alpha(\gamma-\mu) \geq 0$ i.e. $k \geq a(\mu-\gamma)$ then this expression (4.4) is positive.

Thus the theorem about non existence of periodic orbit will hold if the constants $\mathrm{a}, \mu, \gamma, \mathrm{k}$ are positive and $k \geq a(u-\gamma)$.

In general we may expect $\mu>\gamma$ (ideally). Thus for a given population a, $\mu, \gamma$ may be known, so we can choose $\mathrm{k}$ such that $k \geq a(\mu-\gamma)$ and the theorem holds.

We rescale (4.3) by taking

$x=\frac{k}{\beta+\gamma} I_{v} y=\frac{k}{\beta+\gamma} R_{s} \tau=(\beta+\gamma) t$

in order to study the properties of the disease-free equilibrium $\mathrm{E}_{0}$ and the endemic equilibrium $\mathrm{E}^{*}$.

Then we obtain

$$
\begin{aligned}
& \frac{d x}{d \tau}=\frac{x(1+p x)}{1+q x^{2}}(A-x-y)-m x \\
& \frac{d y}{d \tau}=r x-y
\end{aligned}
$$

where

$A=\frac{k \alpha}{\beta(\beta+\gamma)}, \quad p=\frac{a(\beta+\gamma)}{k}, \quad q=\frac{b(\beta+\gamma)^{2}}{k^{2}}, \quad m=\frac{\beta+\mu}{\beta+\gamma}, \quad r=\frac{\mu}{\beta+\gamma}$

Remark: The trivial equilibrium $(0,0)$ of system (eq. (4.5) and eq. (4.6)) is the disease-free equilibrium $\mathrm{E}_{0}$ of model (3.1) and the unique positive equilibrium $\left(\mathrm{x}^{*}, \mathrm{y}^{*}\right)$ of system (eq. (4.5) \& eq. (4.6)) is the endemic equilibrium $\mathrm{E}^{*}$ of model (3.1) if and only if $m-A<0$, where

$$
x^{*}=\frac{-(1+r-A p)+\sqrt{(1+r-A p)^{2}-4(p+r+m q)(m-A)}}{2(p+r+m q)}, \quad y^{*}=r x^{*}
$$

The Jacobian matrix of system (eq. (4.5) \& eq. (4.6)) is

$$
M=\left[\begin{array}{cc}
\frac{A-2 x-y+2 p A x-3 p x^{2}-2 p x y-A q x^{2}+q x^{2} y-p q x^{4}-m-2 m q x-m q^{2} x^{4}}{\left(1+q x^{2}\right)^{2}} & \frac{-x(1+p x)}{1+q x^{2}} \\
r & -1
\end{array}\right]
$$

Now, we determine the stability and topological type of the disease free equilibrium $(0,0)$ of system (eq. (4.5) \& eq. (4.6)).

Theorem 4.3: The disease-free equilibrium $(0,0)$ of system (eq. (4.5) \& eq. (4.6)) is

(i) a stable hyperbolic node if $m-A>0$;

(ii) a saddle-node if $m-A=0$;

(iii) a hyperbolic saddle if $m-A<0$.

Proof. The Jacobian matrix of system (eq. (4.5) \& eq. (4.6)) at $(0,0)$ is

$M_{0}=\left[\begin{array}{cc}A-m & 0 \\ r & -1\end{array}\right]$

The eigen values for $\mathrm{M}_{0}$ are $\lambda_{1}=A-m_{s} \lambda_{2}=-1_{\text {. }}$

For $m-A<0$, one eigen value is positive and another is negative, therefore the disease-free equilibrium $(0,0)$ of system (eq. (4.5) \& eq. (4.6)) is a hyperbolic saddle.

For $m-A>0$, both eigen values are negative, therefore the disease-free equilibrium $(0,0)$ of system (eq. (4.5) \& eq. (4.6)) is a stable hyperbolic node. If $m-A=0$, then there exists a small neighborhood $\mathrm{N}_{0}$ of $(0,0)$ such that the dynamics of system (eq. (4.5) \& eq. (4.6)) are equivalent to that of

$$
\begin{aligned}
& \frac{d x}{d \tau}=(A p-1) x^{2}-x y+O\left((x, y)^{\mathrm{d}}\right) \\
& \frac{d y}{d \tau}=r x-y
\end{aligned}
$$

Since the degree of $\mathrm{x}$ in first term $\mathrm{dx} / \mathrm{d} \tau$ is 2 which is even, $(0,0)$ is a saddle-node (refer theorem 2.11.1 of Perko [12], pp. 150).

This completes the proof.

When $m-A<0$, we discuss the stability and topological type of the endemic equilibrium $\left(\mathrm{x}^{*}, \mathrm{y}^{*}\right)$. The Jacobian matrix of (eq. (4.5) \& eq. (4.6)) at $\left(x^{*}, y^{*}\right)$ is 


$$
\begin{aligned}
& M_{1}=\left[\begin{array}{cc}
\frac{(A p-1)\left(1+q x^{* 2}\right) x^{*}-(r+2)\left(1+q x^{* 2}\right) p x^{* 2}-2 m q x^{*}}{\left(1+q x^{* 2}\right)^{2}} & \left.\frac{-x^{*}\left(1+p x^{*}\right)}{\left(1+q x^{* 2}\right)^{2}}\right] \quad(4.7) \\
\operatorname{det}\left(M_{1}\right)=\frac{-(A p-1)\left(1+q x^{* 2}\right) x^{*}+(r+2)\left(1+q x^{* 2}\right) p x^{* 2}+2 m q x^{*}}{\left(1+q x^{* 2}\right)^{2}}+\frac{r x^{*}\left(1+p x^{*}\right)}{\left(1+q x^{* 2}\right)^{2}}<0
\end{array}\right.
\end{aligned}
$$

Hence, $\operatorname{det} M_{1}>0$ if $A p-1<0$ and $\left(\mathrm{x}^{*}, \mathrm{y}^{*}\right)$ is a node or a focus or a center. We also have the following result on the stability of $\left(\mathrm{x}^{*}, \mathrm{y}^{*}\right)$.

Theorem 4.4: There is a unique endemic equilibrium $\left(\mathrm{x}^{*}, \mathrm{y}^{*}\right)$ of model (eq. (4.5) \& eq. (4.6)), which is a stable node if $m-A<0$.

Proof. The stability of $\left(\mathrm{x}^{*}, \mathrm{y}^{*}\right)$ is determined by $\operatorname{tr}\left(M_{1}\right)$. From (4.7), we have

$$
\operatorname{tr}\left(M_{1}\right)=\frac{(A p-1)\left(1+q x^{* 2}\right) x^{*}-(r+2)\left(1+q x^{* 2}\right) p x^{* 2}-2 m q x^{*}}{\left(1+q x^{* 2}\right)^{2}}-1
$$

If $A p-1<0$ then it implies $\operatorname{tr}\left(M_{1}\right)<0$

This completes the proof.

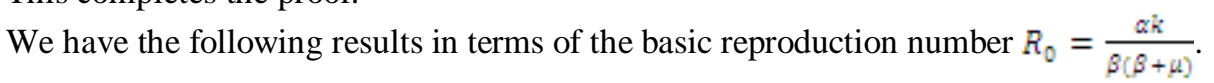

Summarizing theorems 4.2, 4.3 and 4.4, we have the following theorem.

Theorem 4.5:

(i) If $\mathrm{R}_{0}<1$, then model (3.1) has a unique disease-free equilibrium $\mathrm{E}_{0}=(\alpha / \beta, 0,0)$, which is a global attractor in the first octant.

(ii) If $\mathrm{R}_{0}=1$, then model (3.1) has a unique disease-free equilibrium $\mathrm{E}_{0}=(\alpha / \beta, 0,0)$, which attracts all orbits in the interior of the first octant.

(iii) If $\mathrm{R}_{0}>1$, then model (3.1) has two equilibria, a disease-free equilibrium $\mathrm{E}_{0}=(\alpha / \beta, 0,0)$ and an endemic equilibrium $E^{*}=\left(S^{*}{ }_{\mathrm{w}} I_{\mathrm{o}}^{*} R^{*}\right)$. The endemic equilibrium $\mathrm{E}^{*}$ is a global attractor in the interior of the first octant.

\section{Numerical Simulation}

We give the numerical simulation of the solutions of system (2.1) using MATLAB. Fig. 2 shows that $\mathrm{S}(\mathrm{t})$ approaches steady state value whereas $\mathrm{I}(\mathrm{t})$ and $\mathrm{R}(\mathrm{t})$ tends to zero with the evolving time and hence the disease goes to extinct at $\mathrm{R}_{0}<1$. When $\mathrm{R}_{0}>1, \mathrm{~S}(\mathrm{t})$ and $\mathrm{R}(\mathrm{t})$ approach the steady state values while $\mathrm{I}(\mathrm{t})$ increases first and attains a steady state value as shown in Fig. 3 and epidemic occurs.

Variations of $I^{*}$ with varying values of $a$ and $b$, keeping all the other values constant, are shown in Fig. 4 and Fig. 5 respectively. In Fig. 4, it is shown that $I^{*}$ increases with the increase of a and epidemic attains its peak at high values of a. Fig. 5 shows that the value of $I^{*}$ decreases as $b$ increases and it tends to zero with increasing $\mathrm{b}$.

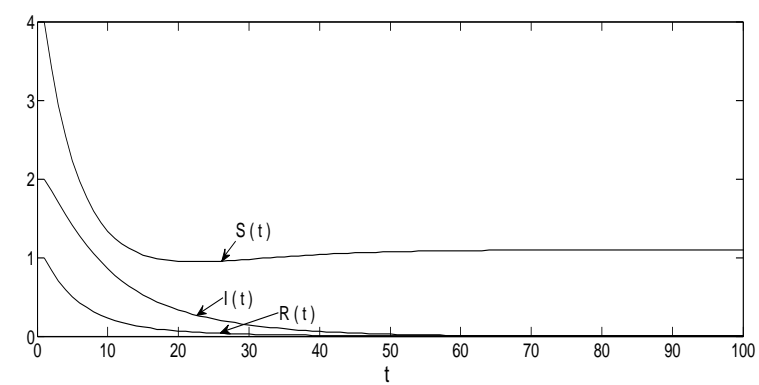

Fig. 2. here $S(0)=4, I(0)=2, R(0)=1, \alpha=0.9, \beta=0.82, \gamma=0.25, k=0.4, a=2.9, b=4.6, \mu=0.11, R_{0}=0.47$

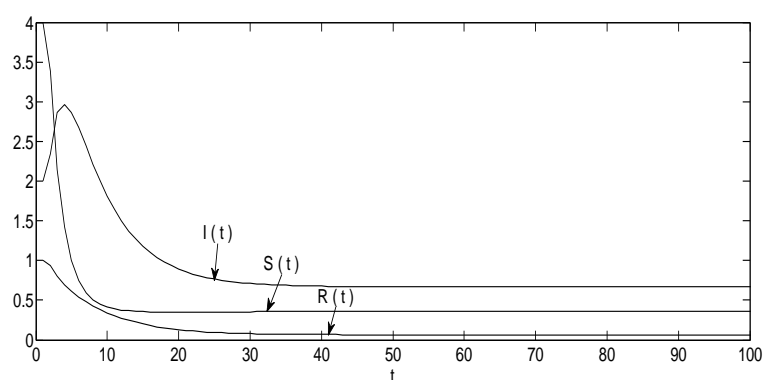

Fig. 3. here $S(0)=4, I(0)=2, R(0)=1, \alpha=0.9, \beta=0.82, \gamma=0.25, k=2.7 a=2.9, b=4.6, \mu=0.11, R_{0}=3.186$ 


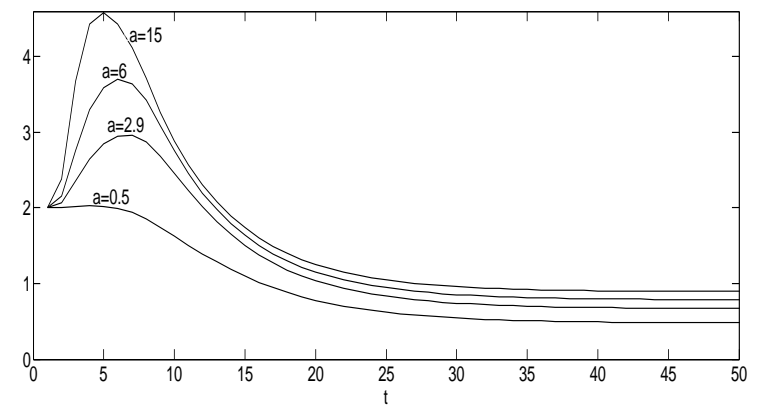

Fig. 4: dependence of $I^{*}$ on a

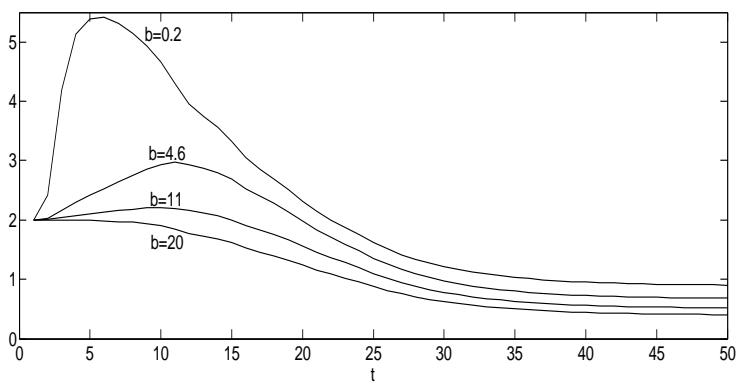

Fig. 5: dependence of $I^{*}$ on $b$

\section{Concluding Remarks}

Using the numerical simulations, we conclude that when $\mathrm{R}_{0} \leq 1$ disease dies out and the disease free equilibrium is globally attractive. When $\mathrm{R}_{0}>1$, the endemic equilibrium is globally stable i.e. epidemic occurs. Also it is seen that though $R_{0}$ does not depend on a and $b, I^{*}$ varies with the variations in a and $b$. It implies that as the superstitions about the disease increases within the population, the number of infectives also increases and the disease persists in the population. While if we create awareness among the population about the disease and the precautions are taken then the disease eradicate significantly

\section{References}

[1]. W.O. Kermack, A.G.McKendrick, Contribution to mathematical theory of epidemics, P. Roy.Soc. Lond. A Mat., 115, 1927, 700721 .

[2]. H. W. Hethcote, The mathematics of infectious diseases, SIAM review, 42, 2000, 599-653.

[3]. V. Capasso, G. Serio, A generalization of the Kermack-McKendrick deterministic epidemic model, Math. Biosci., 42, 1978, 43-61.

[4]. W. Wang, S. Ruan, Simulating the SARS outbreak in Beijing with limited data, J. Theoret. Biol., 227, 2004, $369-379$.

[5]. W. M. Liu, S. A. Levin, Y. Iwasa, Influence of nonlinear incidence rates upon the behavior of SIRS epidemiological models, J. Math. Biol., 23, 1986, 187-204.

[6]. W. R .Derrick, P. van den Driessche, A disease transmission model in a nonconstant population, J. Math. Biol., 31, 1993, 495-512.

[7]. H. W. Hethcote, S. A. Levin, Periodicity in epidemiological models, in:L. Gross, T.G. Hallam, S. A. Levin (Eds.), Applied Mathematical ecology, Springer-Verlag,Berlin, 1989, 193-211.

[8]. H. W. Hethcote, P. van den Driessche, Some epidemiological models with nonlinear incidence, J. Math. Biol., 29, $1991,271-287$.

[9]. M. E. Alexander, S. M. Moghadas, Periodicity in an epidemic model with a generalized non-linear incidence, Math. Biosci., 189, 2004, 75-96.

[10]. D. Xiao, S. Ruan, Global Analysis of an epidemic model with nonmonotone incidence rate, Math Biosci., 208, 2007, 419-429.

[11]. Mimmo Iannelli, The mathematical modeling of epidemics, Mathematical models in Life Sciences: Theory and simulation, 2005.

[12]. L. Perko, Differential Equations and Dynamical Systems (Springer-Verlag, New York, 1996).

[13]. J.N.Kapur, Mathematical Modelling (New Age International Publishers, New Delhi, 1988).

[14]. Herbert W. Hethcote, The Basic Epidemiology Models: Models, Expressions For R ${ }_{0}$, Parameter Estimation, And Applications (etextbook).

[15]. Elizabeth S. Allman, John A. Rhodes, Mathematical Models in Biology, An Introduction (Cambridge University Press, 2004).

[16]. S. Pathak, A. Maiti, G.P. Samanta, Rich dynamics of an SIR epidemic model, Nonlinear Analysis: Modelling and Control, 15, 2010, 71-81.

[17]. Richard Bellman, Stability theory of differential equations (Dover Publications Inc., Newyork, 2008).

[18]. Diekmann O. and Heesterbeek J. A. P., Mathematical epidemiology of infectious diseases (Wiley series in Mathematical and computational biology, Wiley, 2000). 